\title{
Intensive intravenous regime for acute severe colitis
}

\author{
Rupa Banerjee, ${ }^{1}$ Matthew Philip, ${ }^{2}$ Shobna Bhatia ${ }^{3}$
}

\begin{abstract}
Asian Institute of Gastroenterology, Hyderabad ${ }^{1}$

PVS Memoral Hospital,

Kochi Kerala, ${ }^{2}$

Department of Gastroenterology,

Seth G S Medical College and

K E M Hospital, Mumbai ${ }^{3}$
\end{abstract}

Correspondence:

Dr. R. Banerjee

Email: rupabanerjee.aig@gmail.com

\section{ABSTRACT}

Acute severe exacerbation of ulcerative colitis is a potentially life threatening medical emergency. The management of acute severe ulcerative colitis depends on early recognition and prompt initiation of intensive intravenous treatment along with continuous objective monitoring for possible medical failure. The intensive regime is the accepted standard of care. This includes primarily a) intravenous corticosteroids, b) intravenous supportive management, and d) intravenous antibiotics in instances. This review discusses the timing, duration and dosage of the intensive intravenous treatment including the evidence based protocol for effective monitoring to enable timely escalation to second line therapy \& colectomy.

Keywords : intensive regime, acute severe ulcerative colitis

\section{Introduction}

Ulcerative colitis (UC) is a complex disorder with diffuse colonic mucosal inflammation causing recurrent symptoms and morbidity. Acute severe exacerbation of ulcerative colitis (ASC) is a potentially life-threatening medical emergency, and requires prompt and effective management. The lifetime risk of a severe exacerbation is estimated to be around $15 \%$ and is significantly higher in pediatric-onset disease (50-80\% vs. $8-20 \%) .{ }^{1,2,3}$ The management of ASC depends on early recognition and prompt initiation of intensive intravenous treatment along with continuous objective monitoring for possible medical failure.

Prior to the corticosteroid era, a severe attack of UC was associated with high mortality. First year mortality from ASC was found to $75 \%$ and $22 \%$ in studies from Birmingham (1933) and Oxford (1950), respectively. ${ }^{4}$ The landmark work of Truelove in 1955 showed a reduction in mortality from $24 \%$ in the placebo group to $7 \%$ in the steroid-treated group. ${ }^{5}$ A similar efficacy was reported by Lennard-Jones et al. ${ }^{6}$ The mortality rates subsequently came down to $<1 \%$ with timely second-line medical rescue therapy or colectomy. ${ }^{7}$

(C) Tropical Gastroenterology 2014
Today; an intensive regime including intravenous corticosteroids has become the standard of care. However, despite the undisputed improved outcome with such intensive intravenous regime, the dose and duration of treatment are yet to be optimized. This review discusses the intensive intravenous treatment for ulcerative colitis including the evidence-based protocol for effective monitoring of disease progress and treatment escalation when needed.

\section{The time to initiate intensive intravenous treatment}

Acute severe ulcerative colitis, (ASC) is usually defined as per the original classification proposed by Truelove and Witts. ${ }^{5}$ They suggested that six or more bowel motions per day associated with one or more of the following: temperature $>37.8^{\circ} \mathrm{C}$, large amounts of rectal bleeding, heart rate $>90$ beats per minute, hemoglobin $<10.5 \mathrm{~g} / \mathrm{dL}$ or an erythrocyte sedimentation rate $(\mathrm{ESR})>30 \mathrm{~mm} / \mathrm{h}$ indicate severe colitis. These criteria have subsequently been validated and allow 
simple, rapid stratification of ulcerative colitis outpatients. ${ }^{8}$ Unprepared sigmoidoscopy with minimal inflation also provides additional information on disease severity. Endoscopic scoring systems particularly the Baron score have been helpful. ${ }^{9,10}$ It is generally accepted that all patients fulfilling criteria of ASC must be admitted to the hospital and instituted on intensive intravenous treatment.

\section{The intensive intravenous regime}

The intensive intravenous regime for acute severe ulcerative colitis comprises of a) intravenous corticosteroids, b) intravenous supportive and preventive management, c) intravenous nutrition, and d) intravenous antibiotics.

\section{Intravenous corticosteroids}

Intravenous corticosteroids remain the first line of therapy for ASC ever since Truelove and Jewell ${ }^{11}$ published their first trial 1974. Forty-nine patients were treated with intensive intravenous corticosteroids and 36 of 49 (73\%) achieved complete remission by day 5 . The role of IV corticosteroids has been validated and confirmed since than. A systematic review of 32 trials consisting of over 2000 patients treated with corticosteroids for acute severe UC between 1974 and 2006 showed an overall $67 \%$ response rate to steroids. ${ }^{12}$

A number of parenteral corticosteroids, including hydrocortisone, prednisolone, methylprednisolone, bethamethasone, and adrenocorticotropic hormone, have been tried for the treatment of severe UC. No significant difference in response has been observed between the various formulations used. ${ }^{12}$ However, there have been very few trials comparing different treatment regimens. A small study by Meyers et al compared adrenocorticotropic hormone with hydrocortisone. Of the 31 patients who had not received any prior oral corticosteroids, ACTH induced remission in $63 \%$ vs. $27 \%$ with hydrocortisone. However in 35 patients who had received prior corticosteroids, $53 \%$ responded to hydrocortisone vs. $25 \%$ on ACTH. ${ }^{13}$

A daily dose equivalent to $400 \mathrm{mg}$ hydrocortisone $(100 \mathrm{mg}$ four times daily) or $60 \mathrm{mg}$ methylprednisolone is generally given. No studies examining the dose range of the various intravenous corticosteroids has been carried out for ASC. Limited data available does not suggest that higher doses are more effective; higher doses tend to expose the patient to greater side effects. Suboptimal doses are less effective. ${ }^{14}$ A recent meta-analysis of response to corticosteroid treatment controlled for baseline disease severity showed no correlation between corticosteroid dose and colectomy rate, further confirming no significant advantage of increasing the dose beyond $60 \mathrm{mg}$ methylprednisolone per day or equivalent. ${ }^{12}$ Prednisolone or methylprednisolone are preferred at some centers because of less sodium-retention and potassiumwasting.

Bolus administration of corticosteroids as single or daily divided doses is standard practice. No other dosing regimens have been shown to be superior or safer to bolus dosing. Bossa et al randomized 66 patients to receive up to $60 \mathrm{mg}$ methylprednisolone per day by either twice a day bolus or continuous infusion. There were no differences in treatment response or adverse effects between the two groups. About $50 \%$ patients in each group achieved clinical remission by day 7 , and $35 \%$ of the continuous infusion group had undergone elective colectomy by one year compared to $28 \%$ in the bolus group. ${ }^{15}$ Two prospective studies have shown that pulsed therapy can be effective for treatment of UC. ${ }^{16,17}$ However, both these studies used unusually high corticosteroid doses and did not have a control arm. With no obvious difference in terms of efficacy or safety, the selection of a parenteral steroid and its dosing regimen is often based on the physician and center's experience.

\section{Intravenous supportive and preventive therapy}

Intravenous corticosteroids remain the cornerstone of ASC treatment. However supportive management is equally essential. This includes intravenous fluids and electrolytes, blood transfusions and thromboembolism prophylaxis. Majority of hospitalized patients with ASC need intravenous fluids and correction of electrolyte imbalance. Serum potassium is particularly important since hypokalemia predisposes to colonic dilatation. Serum potassium $>4 \mathrm{mmol} / \mathrm{L}$ is considered adequate and most patients need routine potassium replacement at 60 $\mathrm{mmol} / \mathrm{L}$ over $24 \mathrm{~h} .{ }^{18}$ Patients with hemoglobin $<10 \mathrm{~g} / \mathrm{dL}$ should be advised a blood transfusion. This is important because oral iron therapy can aggravate mucosal inflammation induced by oxygen free radicals ('Fenton' reaction) and is best avoided. ${ }^{18,19}$ Antidiarrhoeal agents, anticholinergics and opiates are to be avoided as they can decrease colonic motility thereby increasing the risk of colonic dilatation and toxic megacolon. ${ }^{8,20}$ 
Intravenous heparin and low molecular heparin therapy have been advocated for acute severe colitis to prevent the development of venous thromboembolism. ${ }^{8,21}$ However the risk is age dependant and is much higher in older patients. In a recent analysis, 27 cases of deep vein thrombosis per 10,000 person-years were noted in the 0-20 year age group (95\% CI: 3.4-29.3) vs. 207 per 10,000 in the $>60$ year age group. ${ }^{22}$ Hence routine heparin prophylaxis is perhaps not justified in children. It is important to clarify that numerous meta-analyses have shown that heparin has no role in inducing remission and is not a therapeutic adjunct. ${ }^{23,24}$

\section{Intravenous nutrition}

Three prospective RCTs have compared TPN and bowel rest with either a standard oral diet or enteral nutrition as adjunct therapy with intravenous corticosteroids. There was no difference in the need for colectomy between the groups. ${ }^{25-27}$ There is no evidence that keeping patients on complete bowel rest with total parenteral nutrition or elimination diets improves the course of TPN is associated with complications such as pneumothorax, electrolyte imbalance and sepsis. However, continuous nutritional assessment, including measuring daily weight and calorie counts, is essential for optimization of nutritional status. Short-term parenteral nutrition should be advised if severe nausea and vomiting are present, or in the presence of severe abdominal pain when the patient is unable to tolerate oral diet. Total enteric nutrition with polymeric formula has been found to be safe and well-tolerated in a small prospective cohort of severe UC. ${ }^{28}$ However, the results of enteral administration of formulated foods which is known to work in paediatric Crohn's disease could not be replicated in ASC. Milk and milk products (lactose) restriction is not recommended routinely and is beneficial only in individuals with lactose intolerance. ${ }^{29}$

\section{Intravenous antibiotics}

Several trials have shown that empiric use of intravenous antibiotics along with corticosteroids does not offer any additional benefit over corticosteroid treatment alone. Chapman et al randomized hospitalized patients with ASC to receive intravenous metronidazole $500 \mathrm{mg}$ every 8 hours $(n=19)$ or placebo $(n=20)$ in addition to corticosteroids. The authors found no difference in outcome. ${ }^{30}$ A second study randomized 39 patients to receive metronidazole $500 \mathrm{mg}$ three times a day and tobramycin $4 \mathrm{mg} / \mathrm{kg}$ in divided doses every 8 hours $(\mathrm{n}=19)$ or placebo $(n=20)$ in addition to corticosteroids and found no difference in response. ${ }^{31}$ Another study investigated patients randomized to receive either intravenous ciprofloxacin $400 \mathrm{mg}$ twice daily or placebo for 10 days in addition to corticosteroids. ${ }^{32}$ Like other studies no difference in treatment response was found. However, these studies were underpowered to detect an effect. Antibiotics are indicated in patients who develop signs of sepsis or have a high likelihood of infection due to an impending perforation or toxic megacolon. Metronidazole or vancomycin is also indicated in patients with concurrent C. difficile infection. Empiric antibiotic treatment should also be strongly considered if the patient presents with ASC and stool C. difficile cultures are pending. ${ }^{8,33}$

\section{Duration of intensive intravenous treatment and predicting need for salvage therapy}

Around $30 \%$ of ASC patients will not respond to corticosteroids, and this figure has stayed remarkably constant over the years. ${ }^{7,11,34}$ Also, approximately $80 \%$ of patients who fail corticosteroids require a colectomy within next 10 years regardless of treatment. ${ }^{10}$ All clinical guidelines recommend a second-line salvage therapy in patients with no response to corticosteroids. A timely decision can significantly improve outcome. ${ }^{35,36}$ However the length of time that should be allowed for patients to respond to intravenous therapy is contentious. In 1974, Truelove and Jewell recommended colectomy if there was no response to intravenous corticosteroids after five days. This 5-day rule has been widely adopted. Others however have reported that steroids can be given safely up to 10 days thereby allowing patients more time to respond. ${ }^{37}$ There appears to be no proven benefit of extending IV corticosteroids beyond 10 days. ${ }^{38}$ The decision between colectomy or salvage therapy should be made within the first 3-5 days for a favorable outcome.

Simple clinical and laboratory variables are often used to predict response to corticosteroid therapy. ${ }^{12,39}$ The Travis criterion at day 3 of corticosteroid therapy is often used. Accordingly patients with a stool frequency of more than eight per day or a stool frequency of three per day plus a CRP $>45$ $\mathrm{mg} / \mathrm{dL}$ have a $85 \%$ likelihood of requiring colectomy. ${ }^{34}$ A timebound approach for predicting steroid responsiveness has also been demonstrated in other studies. Lindgren et al prospectively studied admissions with ASC and suggested a mathematical 
model to predict colectomy. A value of (number of stools in 24 hours $+0.143 \mathrm{CRP}(\mathrm{mg} / \mathrm{dL}))>8$ in the model predicted a $72 \%$ colectomy rate. ${ }^{40} \mathrm{Ho}$ and colleagues did a retrospective analysis of ASC and identified certain criteria to score for predicting colectomy or need for rescue therapy. The number of stools (score 1-4), hypoalbuminaemia <30 g/L (score 1) and colonic dilatation $>5.5 \mathrm{~cm}$ (score 4 ) were noted as predictors, with $85 \%$ of patients with a score of 4 or more requiring colectomy or second-line therapy. ${ }^{41}$

For children, the PUCAI (Pediatric UC Activity Index) is the only validated index of UC severity. In a prospective study of 128 hospitalized pediatric patients, the PUCAI scores at days 3 and 5 could predict response to steroids. Patient with PUCAI score $>45$ on day 3 were likely to fail steroids (negative predictive value $>94 \%$ ). A score $>70$ on day 5 reliably guided implementation of salvage therapy with a positive predictive value of $100 \%$ and high specificity. ${ }^{42}$ In addition, two studies have shown endoscopic severity to be a predictor of steroid failure and the need for colectomy, ${ }^{1,43}$ but a full length colonoscopy can increase the risk of perforation and is not recommended.

\section{Conclusion}

Approximately $15 \%$ of patients with UC experience an acute severe attack which requires hospital admission. The intensive intravenous regime is the standard of care for ASC patients. This includes intravenous corticosteroids together with fluid and electrolyte support. Intravenous antibiotics are not recommended unless there is obvious sepsis, impending perforation or toxic megacolon. Intravenous heparin is recommended in adults to prevent thromboembolic complications. A time-bound approach with assessment of response predictors is needed to ensure timely switch to second-line therapy with infliximab, cyclosporine or colectomy. Overall an intensive treatment with early, objective decision, made by an experienced multidisciplinary team for salvage therapy, is the key to successful management of acute severe colitis.

\section{References}

1. Daperno M, Sostegni R, Rocca R, et al. Medical treatment of of severe ulcerative colitis. Aliment Pharmacol Ther. 2004;16(Suppl 4): 7-12.

2. Antoniolli DA. Pediatric inflammatory bowel disease. Pediatr Dev Pathol. 2005;8:2-19.
3. Griffiths AM. Specificities of inflammatory bowel disease in childhood. Best Pract Res Clin Gastroenterol. 2004;18:509-23.

4. Edwards FC and Truelove SC. The Course and Prognosis of Ulcerative Colitis. Gut. 1963;4:299-315.

5. Truelove SC and Witts LJ. Cortisone in ulcerative colitis; final report on a therapeutic trial. Br Med J. 1955;2:1041-8.

6. Lennard-Jones JE, Longmore AJ, Newell AC, Wilson CW and Jones FA. An assessment of prednisone, salazopyrin, and topical hydrocortisone hemisuccinate used as out-patient treatment for ulcerative colitis. Gut. 1960;1:217-22.

7. Rice-Oxley GM, Truelove SC. Ulcerative colitis : course and prognosis. Lancet. 1950;i:663-6.

8. Travis SP, Stange EF, Lemann M, Oresland T, Bemelman WA, Chowers Y, et al. European evidence-based Consensus on the management of ulcerative colitis: Current management. J Crohns Colitis. 2008;2:24-62.

9. Schroeder KW, Tremaine WJ and Ilstrup DM. Coated oral 5aminosalicylic acid therapy for mildly to moderately active ulcerative colitis. A randomized study. $N$ Engl J Med. 1987;317:1625-9.

10. Jakobovits SL and Travis SP. Management of acute severe colitis. Br Med Bull. 2005;75-76:131-44.

11. Truelove SC and Jewell DP. Intensive intravenous regimen for severe attacks of ulcerative colitis. Lancet. 1974;1:1067-70.

12. Turner D, Walsh CM, Steinhart AH and Griffiths AM. Response to corticosteroids in severe ulcerative colitis: a systematic review of the literature and a meta-regression. Clin Gastroenterol Hepatol. 2007;5:103-10.

13. Meyers S, Lerer PK, Feuer EJ, Johnson JW and Janowitz HD. Predicting the outcome of corticoid therapy for acute ulcerative colitis. Results of a prospective, randomized, double-blind clinical trial. J Clin Gastroenterol. 1987;9:50-4.

14. Rosenberg W, Ireland A and Jewell DP. High-dose methylprednisolone in the treatment of active ulcerative colitis. $J$ Clin Gastroenterol. 1990;12:40-1.

15. Bossa F, Fiorella S, Caruso N, Accadia L, Napolitano G, Valvano MR, et al. Continuous infusion versus bolus administration of steroids in severe attacks of ulcerative colitis: a randomized, double-blind trial. Am J Gastroenterol. 2007;102:601-8.

16. Oshitani N, Kitano A, Matsumoto $\mathrm{T}$ and Kobayashi K. Corticosteroids for the management of ulcerative colitis. $J$ Gastroenterol. 1995;30 Suppl 8:118-20.

17. Sood A, Midha V, Sood N and Awasthi G. A prospective, openlabel trial assessing dexamethasone pulse therapy in moderate to severe ulcerative colitis. J Clin Gastroenterol. 2002;35:328-31.

18. Gulliford SR and Limdi JK. Acute severe ulcerative colitis: timing is everything. Postgrad Med J. 2011;87:215-22.

19. Gasche C, Berstad A, Befrits R, Beglinger C, Dignass A, Erichsen $\mathrm{K}$, et al. Guidelines on the diagnosis and management of iron deficiency and anemia in inflammatory bowel diseases. Inflamm Bowel Dis. 2007;13:1545-53.

20. Kornbluth A, Sachar DB and Practice Parameters Committee of the American College of Gastroenterology. Ulcerative colitis practice guidelines in adults: American College Of Gastroenterology, Practice Parameters Committee. Am J Gastroenterol. 2010;105:501-23.

21. Grainge MJ, West J and Card TR. Venous thromboembolism 
during active disease and remission in inflammatory bowel disease: a cohort study. Lancet. 2010;375:657-63.

22. Bernstein CN, Blanchard JF, Houston DS and Wajda A. The incidence of deep venous thrombosis and pulmonary embolism among patients with inflammatory bowel disease: a populationbased cohort study. Thromb Haemost. 2001;85:430-4.

23. Chande N, McDonald JW and Macdonald JK. Unfractionated or low-molecular weight heparin for induction of remission in ulcerative colitis. Cochrane Database Syst Rev. 2008:CD006774.

24. Brown SR, Haboubi N, Hampton J, George B, Travis SP and ACPGBI. The management of acute severe colitis: ACPGBI position statement. Colorectal Dis. 2008;10 Suppl 3:8-29.

25. McIntyre PB, Powell-Tuck J, Wood SR, Lennard-Jones JE, Lerebours E, Hecketsweiler P, et al. Controlled trial of bowel rest in the treatment of severe acute colitis. Gut. 1986;27:481-5.

26. Dickinson RJ, Ashton MG, Axon AT, Smith RC, Yeung CK and Hill GL. Controlled trial of intravenous hyperalimentation and total bowel rest as an adjunct to the routine therapy of acute colitis. Gastroenterology. 1980;79:1199-204.

27. Gonzalez-Huix F, Fernandez-Banares F, Esteve-Comas M, AbadLacruz A, Cabre E, Acero D, et al. Enteral versus parenteral nutrition as adjunct therapy in acute ulcerative colitis. Am J Gastroenterol. 1993;88:227-32.

28. Klaassen J, Zapata R, Mella JG, Aguayo G, Alvarado D, Espinosa $\mathrm{O}$, et al. [Enteral nutrition in severe ulcerative colitis. Digestive tolerance and nutritional efficiency]. Rev Med Chil. 1998;126:899-904.

29. Wright $\mathrm{R}$ and Truelove SC. A Controlled Therapeutic Trial of Various Diets in Ulcerative Colitis. Br Med J. 1965;2:138-41.

30. Chapman RW, Selby WS and Jewell DP. Controlled trial of intravenous metronidazole as an adjunct to corticosteroids in severe ulcerative colitis. Gut. 1986;27:1210-2.

31. Mantzaris GJ, Hatzis A, Kontogiannis P and Triadaphyllou G. Intravenous tobramycin and metronidazole as an adjunct to corticosteroids in acute, severe ulcerative colitis. Am J Gastroenterol. 1994;89:43-6.

32. Mantzaris GJ, Petraki K, Archavlis E, Amberiadis P, Kourtessas $\mathrm{D}$, Christidou A, et al. A prospective randomized controlled trial of intravenous ciprofloxacin as an adjunct to corticosteroids in acute, severe ulcerative colitis. Scand J Gastroenterol. 2001;36:971-4.

33. Carter MJ, Lobo AJ, Travis SP; IBD Section, British Society of Gastroenterology. Guidelines for the management of inflammatory bowel disease in adults. Gut. 2004;53 Suppl 5:V1-16.

34. Travis SP, Farrant JM, Ricketts C, Nolan DJ, Mortensen NM, Kettlewell MG, et al. Predicting outcome in severe ulcerative colitis. Gut. 1996;38:905-10.

35. Randall J, Singh B, Warren BF, Travis SP, Mortensen NJ and George BD. Delayed surgery for acute severe colitis is associated with increased risk of postoperative complications. Br J Surg. 2010;97:404-9.

36. Roberts SE, Williams JG, Yeates D and Goldacre MJ. Mortality in patients with and without colectomy admitted to hospital for ulcerative colitis and Crohn's disease: record linkage studies. BMJ. 2007;335:1033.

37. Chang JC and Cohen RD. Medical management of severe ulcerative colitis. Gastroenterol Clin North Am. 2004;33:235-50, viii.

38. Lichtenstein GR, Abreu MT, Cohen R, Tremaine W; American Gastroenterological Association. American Gastroenterological Association Institute technical review on corticosteroids, 